Institute of $\mathbf{F}_{\text {ood and }} \mathbf{A}_{\text {gricultural }} \mathbf{S}_{\text {ciences }}$

\title{
Manual de los Reglamentos del Agua de Florida: Agencias de Contacto ${ }^{1}$
}

Michael T. Olexa, Laura Minton, Dulcy Miller, y Sarah Corbett ${ }^{2}$

\section{Agradecimientos}

Los autores agradecen a Richard Budell de la Oficina de Política del Agua Agrícola del Departamento de Agricultura y Servicios al Consumidor de Florida. Los autores también agradecen a David H. Hammonds, Consultor del Programa de Salud Ambiental, Oficina de Programas de Aguas de Drenaje en el Sitio, del Departamento de Salud de Florida, y a Edward A. Bettinger, Consultor del Programa de Salud Ambiental, Oficina de Programas de Agua del Departamento de Salud de Florida.

\section{Agencias de Florida}

\section{S-1. Distritos de Manejo de Agua}

Distrito de Manejo de Agua del Suroeste de Florida

2379 Broad Street

Brooksville, FL 34609-6899

(352) 796-7211

(352) 754-6874 (Fax)

1. Este es el documento EDIS FE090, una publicación del Department of Food and Resource Economics, Florida Cooperative Extension Service, Institute of Food and Agricultural Sciences, University of Florida, Gainesville, FL. Publicada Noviembre 2002. Por favor visite la página electrónica EDIS en http://edis.ifas.ufl.edu.

2. Michael T. Olexa, es profesor del Department of Food and Resource Economics, Florida Cooperative Extension Service, Institute of Food and Agricultural Sciences, University of Florida, Gainesville, FL; y miembro de Florida Bar; Presidente de Agricultural Law Committee of The Florida Bar; y Director del Agricultural Law Center. Laura Minton, Dulcy Miller, y Sarah Corbett son estudiantes graduadas de Levin College of Law, University of Florida, Gainesville, FL. Filiberto Reyes-Villanueva fue el traductor de la versión en ingles al español.

Esta publicación esta diseñada para proporcionar información precisa, actualizada y autorizada sobre esta material. Sin embargo, ya que las leyes, reglas administrativas y decisiones de la corte, sobre las cuales están basados, están sujetas a revisión constante; algunas partes de esta publicación podrían ser obsoletas en cualquier momento. Esta publicación es distribuida bajo el entendimiento que los autores no están involucrados en ninguna representación legal u otros servicios profesionales, y que la información contenida aquí no debe ser considerada como un substituto de una asesoria legal. Esta publicación no esta completa en proporcionar toda la información para lograr el cumplimiento de las leyes y reglamentos que gobiernan la protección del agua. Por estas razones, el uso de estos manuales por cualquier persona constituye un acuerdo para mantener libre de daño a los autores, al Florida Cooperative Extension Service, al Institute of Food and Agricultural Sciences, y a la University of Florida por cualquier demanda por responsabilidad de daños, o gastos en que pueda incurrir cualquier persona, como un resultado de hacer referencia o confianza sobre la información contenida en esta publicación. Esta publicación fue apoyada financieramente por el Florida Department of Agriculture and Consumer Services.

El Instituto de Alimentos y Ciencias Agrícolas es Un empleador que opera bajo Acción Afirmativa y provee Oportunidades Igualitarias, dedicado a promocionar la investigación, a información educativa y otros servicios, únicamente a los individuos e instituciones que operan baj discriminación sin considerar color, raza, sexo, edad, incapacidad u origen. Para más información sobre como obtener otras publicaciones de la extensión, comuníquese con la oficina de Servicio de Extensión de su condado. Servicio de Extensión de la Florida / Instituto de Alimentos y Ciencias Agrícolas / Universidad de la Florida / Christine Taylor Waddill, Decana. 
Distrito del Manejo de Agua del Sur de

Florida

3301 Gun Club Road

Post Office Box 24680

West Palm Beach, FL 33416-46680

(800) 432-2045

(561) 686-8800

(561) 687-6276 (Fax)

Distrito de Manejo de Agua de St. Johns

River

Highway 100 West

Post Office Box 1429

Palatka, FL 32177

(386) 329-4500

Distrito de Manejo de Agua de Suwannee

River

Route 3, Box 64

Live Oak, FL 32060

(386) 362-1001

(386) 362-1056 (Fax)

Distrito de Manejo de Agua del Noroeste de

Florida

Route 1, Box 3100

Havana, FL 32060
(850) 539-5999

(850) 539-4380 (Fax)

\section{S-2. Departamento de Protección Ambiental}

Departamento de Protección Ambiental de Florida

3900 Commonwealth Blvd.

Tallahassee, FL 32399-3000

(850) 488-1554

División de Servicios Administrativos y Técnicos

3900 Commonwealth Blvd.

Tallahassee, FL 32399-3000

(850) 488-2955

División de Manejo de Recursos del Aire 3900 Commonwealth Blvd.

Tallahassee, FL 32399-3000

(850) 488-0114

División de Permisos de Recursos

Ambientales

2600 Blairstone Road

Tallahassee, FL 32399-2400

(850) 488-3177

División de Manejo de Desechos 
3900 Commonwealth Blvd.

Tallahassee, FL 32399-3000

(850) 487-3299

División de Instalaciones de Agua

3900 Commonwealth Blvd.

Tallahassee, FL 32399-3000

(850) 487-1855

\section{S-3. Comisión de Caza y Pesca de Agua Dulce}

Comisión de Caza y Pesca de Agua Dulce de Florida

101 Farris Bryant Building

620 South Meridian Street

Tallahassee, FL 32399-1600

(850) 488-3831

\section{S-4. Departamento de Agricultura y Servicio al Consumidor}

Departamento de Agricultura y Servicio al

Consumidor de Florida

Mayo Building, 2nd Floor

The Capitol

Tallahassee, FL 32399-0800

(800) 435-7352

(850) 488-3022

(850) 488-7585 (Fax)

División de Estándares
3125 Conner Boulevard

Tallahassee, FL 32399-1650

(850) 488-0645

División de Bosques

3125 Conner Boulevard

Tallahassee, FL 32399-1650

(850) 488-4274

División de Agricultura y Servicios

Forestales

3125 Conner Boulevard

Tallahassee, FL 32399-1650

(850) 488-3731

\section{S-5. Departamento de Salud}

Departamento de Salud de Florida

4052 Bald Ciprés Way

Tallahassee, FL 32399-1701

(850) $245-4321$

\section{S-6. Departamento de Trabajo y Seguridad del Empleo}

Abolió Junio 30, 2002.

\section{Agencias Nacionales y Números de Teléfonos de la APA}

\section{F-1. Linea Directa para Agua Potable de Consumo}

Proporciona información sobre los reglamentos y programas de la APA sobre agua potable. 
Lunes - Viernes, 8:30 AM - 5:00 PM, EST

Llame gratis, 1 (800) 426-4791

\section{F-2. Linea Directa para las ARCR/ARCRAC (funperfondo)}

Proporciona información sobre la ARCR y ARCRAC y responde a solicitudes de documentos.

Lunes - Viernes, 9:00 AM - 6:00 PM, EST

Llame gratis al, 1 (800) 424-9346

F-3. Planificación de Emergencias y Derecho a Saber de la Comunidad (PEDSC)

Proporciona información acerca de la planificación y preparación que involucran liberaciones de químicos tóxicos

Lunes - Viernes, 9:00 AM - 6:00 PM, EST

Llame gratis al, 1 (800) 424-9346

\section{F-4. Servicio de Información y Ayuda a la ACST}

Proporciona información acerca de las sustancias toxicas y cumplimiento con los reglamentos del ACST. También provee publicaciones y otros materiales que ayudan al cumplimiento.

Lunes- Viernes, 8:30 AM - 5:00 PM, EST

(202) 554-1404

\section{F-5. Red Nacional de Telecommunicaciones en Plaguicidas}

Proporciona información sobre plaguicidas y envenenamientos con plaguicidas.

Las 24 horas del día, 365 días al año. Llame gratis al, 1 (800) 858-7378
F-6. El Administrador en Florida para Registros de Plaguicidas de la APA

Oficina de Plaguicidas/ División de Inspección

Departamento de Agricultura y Servicio al Consumidor

3125 Conner Blvd.., MD-2

Tallahassee, FL. 32399-1650

(850) 4872130

\section{Números dentro de la Región 4 de la APA (Incluye Florida)}

\section{L-1. Información General para la Region 4}

345 Courtland Street, NE

Atlanta, GA 30365

(800) 241-1754

(Se pueden mandar preguntas escritas a esta dirección)

\section{L-2. Sección de Cumplimiento de Desechos Peligrosos}

Supervisa el cumplimiento con ARCR y resuelve los problemas de los ciudadanos respecto a producción de desechos peligrosos.

(404) 5628579

\section{L-3. Sección de Aguas Subterráneas}

Se relaciona con la Contaminación de Aguas Subterráneas, incluyendo las violaciones de AACS

(404) 652-9450 


\section{L-4. Rama del Superfondo para Emergencias}

Respuestas de manejo a derrames y otros puntos de ARCR/ ARCRAC

(800) 424-9346

404) 562-8700 para derrames

\section{L-5. Funcionamiento e Instalaciones del Acta de Agua Limpia}

Rama/ Sección de Cumplimiento

Se relaciona con el cumplimiento del Acta

del Agua Limpia

(404) 562-9345

Sección de Permisos y Aplicación de Agua

Permisos: (404) 562-9304

Aplicación: (404) 562-9770 